\title{
A psicologia médica no centro de tratamento intensivo do Hospital Universitário Pedro Ernesto
}

\author{
Psychology in Pedro Ernesto University Hospital intensive care unit
}

Janete A. Araújo*

Elizabeth M. P. Leitão

\section{Resumo}

A unidade de tratamento intensivo (UTI) é um ambiente de cuidados da saúde com potencial de gerar estados emocionais que podem interferir na evolução do paciente. Os cuidados são oferecidos, através de tratamentos complexos, aos pacientes em graves condições médicas, a fim de garantir a sua sobrevivência e a melhoria do seu estado geral. Além da debilidade física, os pacientes são, ainda, submetidos a situações de estresse importante. Frequentemente mantidos sedados durante o período de recuperação da consciência, são expostos a inúmeros fatores extremamente difíceis do ponto de vista emocional, acarretando síndromes psiquiátricas bastante prevalentes e atenção especializada. A hospitalização de um membro da família desorganiza a dinâmica familiar, ficando ainda mais complexa se a internação ocorre na UTI, pois esta, por si só, simboliza o risco iminente de morte. Os problemas derivados das características ambientais da unidade, cuidado ao paciente, necessidade de uma sólida base de conhecimentos e dificuldades de comunicação interpessoal aumentam a sobrecarga emocional na equipe de saúde. Provocam ansiedade, depressão e o aparecimento de mecanismos de defesa para lidar com as situações de conflito. A inserção do psicólogo neste ambiente objetiva identificar e atuar sobre aspectos que possam interferir na adaptação do paciente ao período de hospitalização e na sua recuperação. A equipe de psicologia médica da UTI geral do Hospital Universitário Pedro Ernesto visa identificar e apoiar os aspectos emocionais dos pacientes e familiares, contribuir na adequada evolução desses indivíduos, bem como promover uma boa comunicação entre as pessoas envolvidas no processo, visando melhorar as relações e o bem-estar de todos.

Descritores: Unidades de terapia intensiva; $\mathrm{Pa}$ cientes; Comunicação; Equipe de assistência ao paciente; Psicologia médica. 


\section{Abstract}

The intensive care unit (ICU) is an environment for health care able to generate emotional situations that can intervene in the patient evolution. Care is offered through complex treatments to patients that present serious medical situation, in order to assure the survival and to improve better health conditions. Besides their physical weakness patients are submitted to important stress. Some are often maintained in a state of sedation and, during the period of progressive recovery of consciousness, they are exposed to various extremely difficult factors from an emotional point of view, causing psychiatric syndromes quite prevalent and requiring specialized attention. The hospitalization of a family member disrupts the family dynamic, addittionally, if hospitalization occurs in the ICU, the situation is even more complex because the ICU itself symbolizes the imminent risk of death. The problems that come from the environmental characteristics of the unit, the patient care, the need for a solid basis of knowledge and the difficulties on interpersonal communication increase the emotional charge on the healthcare team. This causes anxiety responses, depression and the development of defense mechanisms to deal with conflict situations. The introduction of the psychologist in this environment aims to identify and act on aspects that can intervene on the patient adaptation in the hospitalization period and his recuperation. Medical Psychology Service of General ICU of the Pedro Ernesto University Hospital aims at identifying and supporting emotional aspects of patients and families in order to contribute to a proper evolution of these individuals, as well as to promote a good communication between people involved in the process, in order to improve the better relations and the comfort of everybody.

Keywords: Intensive care units; Patients; Communication; Patient care team; Psychology, medical.

\section{Introdução}

A inserção da psicologia nos hospitais brasileiros aconteceu em um período recente da história do país, de acordo com Angerami-Camon, ${ }^{1}$ no início dos anos 1950. No centro de tratamento intensivo (CTI) geral do Hospital Universitário Pedro Ernesto (HUPE), desde a década de 1990 a psicologia médica tem feito parte da equipe multiprofissional, buscando dar suporte psicológico aos pacientes e seus familiares, facilitando o enfrentamento dos momentos de crise e das adaptações em relação ao processo de internação.

Quando o paciente é admitido numa unidade fechada fica exposto a diversos agentes estressores: confinamento, restrição ao leito, cirurgias, uso de aparelhos, ruídos e iluminação constantes, realização frequente de exames, afastamento do trabalho, dos amigos, dificuldade em conciliar o sono e mudanças alimentares repentinas, para citar apenas alguns. Esses agentes podem ocasionar reações emocionais em variados níveis, como ansiedade, medo, conflitos, insegurança, irritabilidade, dentre outras comumente relacionadas ao contexto da internação. ${ }^{2}$

$\mathrm{Na}$ unidade de terapia intensiva (UTI), a rotina de trabalho mais acelerada, o clima constante de apreensão e as situações de morte iminente tornam ainda mais possíveis as interferências emocionais da hospitalização no paciente e em seus familiares.

A preocupação generalizada dos profissionais da área de saúde em prestar uma assistência mais humanizada reforça a necessidade de manejar de forma mais adequada os aspectos emocionais do adoecer. Dessa forma, os profissionais de saúde estão sendo estimulados a se aperfeiçoarem e a complementarem seus conhecimentos para que possam atender aos pacientes a partir uma visão biopsicossocial, não mais apenas biológica. ${ }^{3}$ Trata-se de uma mudança difícil, que necessita da ajuda do psicólogo.

O objetivo deste artigo é descrever a atuação dos profissionais de psicologia no CTI geral do HUPE, citando os procedimentos e recursos uti- 
lizados, nas intervenções com todos os sujeitos envolvidos no processo de internação: pacientes, familiares e equipe de saúde.

\section{Unidade de terapia intensiva}

No final da década de 1940, início da de 1950, uma grande epidemia de poliomielite assolou a Europa e a América do Norte. Com o objetivo de proporcionar a necessária assistência ventilatória aos pacientes mais graves, foram criadas, nos Estados Unidos, as unidades de assistência ventilatória que dariam origem posteriormente à UTI ou CTI.

A UTI, destinada ao atendimento de pacientes em risco de vida, com potencial de recuperação, surgiu, assim, nos Estados Unidos, na década de 1950, época em que foram desenvolvidos os primeiros respiradores artificiais. ${ }^{4}$

No Brasil, esse tipo de serviço surgiu a partir da década de 1960, em hospitais de grande porte no Sul e Sudeste do país. No HUPE, o CTI geral foi inaugurado em abril de 1974.

A UTI foi criada e permanece até hoje com o objetivo de concentrar três componentes críticos: os doentes mais graves com possibilidade de recuperação; o equipamento técnico mais caro e sofisticado; e a equipe com conhecimento e experiência para cuidar desses pacientes e lidar com essa aparelhagem específica. ${ }^{5}$

Nos primeiros anos de sua existência, a aparelhagem e os medicamentos para suporte de vida eram precários, sendo as taxas de mortalidade muito elevadas. Mais tarde, com o avanço tecnológico caracterizado, sobretudo, pelos respiradores e monitores mais modernos e drogas cada vez mais poderosas, a prática da terapia intensiva tornou-se mais eficaz, com acentuado aumento nas taxas de sobrevivência dos pacientes. ${ }^{6}$

Nas décadas de 1980 e 1990, começou a surgir a preocupação com a humanização da prática médica e este conceito passou a ter importância em várias UTIs, inicialmente através da colocação de símbolos externos (abertura de janelas com vista para o exterior, colocação de relógios e calendários, meios de lazer como rádios e televisões etc.) para diminuir o desconforto dos pacientes e, logo a seguir, com um movimento que visava conscientizar os profissionais que ali atuavam a respeito da necessidade de mudanças de atitude na relação com os pacientes e familiares. Talvez a melhora das condições técnicas, com a diminuição do estresse da equipe, tenha sido um importante fator para possibilitar este movimento, ainda em andamento, de humanização das UTIs.

Se por um lado a tecnologia tem contribuído para os avanços da medicina, por outro, de certo modo, tem restringido o contato humano entre a equipe de saúde, os pacientes e familiares.

Nos últimos anos, alguns aspectos vêm contribuindo para mudanças no ambiente das UTIs. Essas mudanças estão relacionadas com a evolução conceitual do processo saúde-doença que implica a interlocução de novos saberes para sua compreensão e abordagem. ${ }^{7}$ Desse modo, hoje se observam equipes mistas, compostas por diversos profissionais da área da saúde, com conhecimentos e habilidades diferenciadas, desenvolvendo um trabalho multidisciplinar.

A equipe multidisciplinar é composta por médicos intensivistas, enfermeiros, fisioterapeutas, técnicos de enfermagem, nutricionistas, assistentes sociais, psicólogos e fonoaudiólogos que possuem treinamento específico e diferenciado voltado para o trabalho com pacientes críticos.

De acordo com Ayres, ${ }^{7}$ a UTI é um lugar de cuidados, onde os atores (profissionais de saúde, pacientes e familiares) precisam estabelecer um padrão de comunicação em que todos compreendam objetivamente o que está sendo dito. As habilidades de comunicação precisam ser eficazes para que se produza alta qualidade de cuidados para o paciente e suas famílias.

As características intrínsecas à UTI, como a rotina de trabalho acentuada, o clima de constante apreensão e as situações de morte iminente, acabam por exacerbar o estado de estresse e tensão, no qual, tanto o paciente quanto a equipe, vivem 24 horas por dia. Esses aspectos, somados à dimensão individual do sofrimento 
da pessoa internada, tais como a dor, o medo, o desconhecido, o isolamento do mundo, desencadeiam vários e fortes fatores psicológicos ${ }^{8}$ que interatuam muitas vezes na enfermidade que o sujeito possui.

\section{A psicologia na UTI}

Ao longo do desenvolvimento da psicologia nota-se que, apesar de um tradicional panorama clínico, houve uma série de mudanças tanto com relação às competências dos psicólogos como à própria visão dos sujeitos dos cuidados psicológicos. ${ }^{9}$ Por volta da década de 1970 iniciaram-se demandas por trabalhos psicológicos sob uma nova perspectiva, que abrange uma clientela diferenciada, um local distinto e, principalmente, a necessidade de se considerar o contexto no qual os sujeitos estão inseridos.

Tudo isto conduziu a uma mudança na concepção da prática, resultando na ampliação do campo. Dentro deste movimento, o que mais se destacou foi o relativo à área da saúde.

Em se tratando da atuação do psicólogo em uma UTI, seu objetivo é dar suporte psicológico, através de uma escuta diferenciada, ao paciente, aos familiares e a toda a equipe de saúde que está envolvida dentro do processo de adoecimento. Possibilita assim a exteriorização e o enfrentamento do sofrimento, da dor e de questões emergentes, facilitando o esclarecimento de dúvidas e de "fantasias" que provocam desconforto.

Ismael ${ }^{10}$ aponta a complexidade do processo vivenciado por estes sujeitos, pois a UTI é vista por algumas pessoas como "local para morrer". Surgem então sentimentos relacionados à desagregação e ao desamparo; o paciente, distante de seus familiares, em um ambiente impessoal, com um aparato tecnológico desconhecido, poderá reagir emocionalmente de forma negativa.

No CTI geral do HUPE, desde a década de 1990, a psicologia médica tem feito parte da equipe multiprofissional, buscando dar suporte psicológico aos pacientes e seus familiares, facilitar o enfrentamento dos momentos de crise e das adaptações em relação ao processo de internação. Estando presente junto ao mé- dico no momento em que são transmitidas aos familiares notícias sobre o estado clínico do paciente, visa mediar a comunicação e dar apoio às duas partes.

Uma característica ímpar do CTI geral do HUPE é a autorização para a visita de crianças aos familiares internados com a permissão dos responsáveis, após avaliação da equipe e desde que acompanhadas da psicóloga.

É função também da psicologia médica facilitar a relação interpessoal entre a equipe multiprofissional, os pacientes e os seus familiares, bem como fornecer espaço para atendê-los sempre que necessário.

\section{O paciente}

Simonetti ${ }^{11}$ afirma que, diante da doença, o ser humano manifesta subjetividades: sentimentos, desejos, pensamentos e comportamentos, fantasias e lembranças, crenças, sonhos, conflitos e sua maneira própria de adoecer.

Esses aspectos podem aparecer como causa da doença, como desencadeador do processo patogênico, como agravante do quadro clínico, como fator de manutenção do adoecimento ou, ainda, como consequência deste. Nesse sentido, o objetivo da psicologia é a elaboração simbólica do adoecimento, ou seja, ajudar o paciente a atravessar a experiência do adoecimento através de sua subjetividade.

O sofrimento físico e psíquico do paciente precisa ser entendido como uma coisa única, pois os dois aspectos interferem um no outro. Através do trabalho da psicologia, o doente pode encontrar um melhor caminho para o enfrentamento da dor, do sofrimento e até mesmo de uma morte mais digna e menos sofrida. É importante criar as condições de comunicação nesse momento: o psicólogo deve buscar o "falar" do paciente, seja através de gestos, olhares ou gemidos, e ser seu porta-voz. ${ }^{12}$

A necessidade de um atendimento psicológico pode não ser percebida pelo paciente, pois suas preocupações estão dirigidas para o corpo e para as ações exploratórias e terapêuticas que nele são praticadas. Atribui seu mal-estar emo- 
cional às vivências oriundas deste corpo doente. ${ }^{6}$

As possíveis reações emocionais do paciente envolvem passividade ou agressividade, argumentação sobre aspectos sem importância, manifestações de raiva ou depressão pela dificuldade em aceitar não só sua doença, mas todo o processo de hospitalização e tratamento. Há também o medo da invalidez permanente, da dependência, da dor física, das alterações na autoimagem, da anestesia, em casos de cirurgia, e do próprio retorno para casa após a hospitalização. ${ }^{10} \mathrm{O}$ paciente, enquanto hospitalizado, costuma ficar mais introspectivo e reavaliar sua vida e seus valores.

Rincon e colaboradores ${ }^{13}$ identificaram em pacientes adultos de UTI índices elevados de transtornos psiquiátricos pré-existentes ou desencadeados/agravados pela doença (delirium: 7,3\%; depressão: $13,7 \%$; ansiedade: $24 \%$; e abuso de álcool: 37,9\%). Estes transtornos podem ser tratados por meio de intervenções psiquiátricas associadas a acompanhamento psicológico.

As questões psicológicas a serem abordadas devem ser focais, visando sempre àqueles aspectos estritamente relacionados à doença, às dificuldades adaptativas à instituição hospitalar, ao processo do adoecer e aos procedimentos diagnósticos. É importante ressaltar que, na UTI, ao contrário do que ocorre habitualmente, é o psicólogo quem procura o paciente, oferecendo-lhe ajuda e ficando disponível também para sua família. ${ }^{10,14}$

\section{O trabalho da psicologia com a família}

O impacto diante do adoecimento e internação de um familiar altera a forma de viver tanto do sujeito que está doente como de seus familiares, pois produz uma interrupção brusca do cotidiano. Isto altera toda a dinâmica familiar e gera sentimentos de insegurança, medo e incerteza. ${ }^{15}$

A família, angustiada e sofrida, que se sente impotente e assustada mediante a ameaça da morte, precisa da atenção do psicólogo, devendo ser envolvida no trabalho com o paciente, pois o vínculo com os familiares é, muitas vezes, uma das poucas motivações que ele tem para enfrentar o sofrimento. O psicólogo deve facilitar, criar e garantir a comunicação efetiva e afetiva entre paciente/família e equipe, identificando qual membro da família tem melhores condições intelectuais e emocionais para receber as informações sobre o doente. ${ }^{12,14}$

Ismae ${ }^{10}$ alerta para o fato de que muitos familiares podem expressar a necessidade de ver o paciente repetidas vezes. Isto pode indicar uma forma da família tentar reconstruir o vínculo quebrado com seu familiar e/ou um meio de lidar com a angústia pela falta do controle que ocorre quando o paciente está longe de seu campo visual.

No CTI do HUPE, desde sua inauguração, sempre foi permitida a visita diária de familiares, sem restrição de número (diferentemente do que ocorre em muitas UTIs) e, após a visita, o médico dá as notícias sobre o estado de seu paciente. Em situações particulares, nas quais um parente não possa comparecer na hora marcada (como em caso de trabalho), é sempre autorizada a visita num horário compatível. No entanto, em virtude da necessidade do paciente ser submetido a algum procedimento, nem sempre é possível permitir a entrada do familiar no horário previsto, causando nesta pessoa um elevado nível de angústia e ansiedade, uma vez que ela não está ciente destes acontecimentos.

De acordo com Botega, ${ }^{16}$ em um estudo realizado na França com 357 familiares de pacientes em UTI, observou-se que 35\% dos membros da família apresentavam depressão e $73 \%$ ansiedade.

Oliveira e Sommerman ${ }^{15}$ afirmam que uma das maiores fontes geradoras de ansiedade é a falta de informação ou informação excessiva e contraditória.

Os familiares precisam ser mantidos informados sobre o que acontece. Uma das maiores necessidades da família é o estabelecimento de uma comunicação clara, honesta e frequente com os membros da equipe que cuida do paciente. $^{17}$ 
É importante, segundo Santos, ${ }^{17}$ "que o profissional mostre atenção, empatia e carinho com o comportamento e sinais não verbais. A expressão facial, o contato visual, a distância adequada e o toque nas mãos, braços ou ombros ajudam a demonstrar empatia e oferecer apoio e conforto".

\section{Trabalho com grupo de familiares}

Em virtude da necessidade de aprimorar a atenção à família surgiu, no CTI geral do HUPE, em 1995, o grupo de informação e apoio. O objetivo deste grupo é promover um espaço para acolher medos, dúvidas e angústias dos familiares de pacientes. Por se tratar de um grupo terapêutico, torna-se possível uma melhor integração, compartilhamento de sentimentos, dúvidas e informações, pois é a partir das trocas realizadas ali, pela visão de outros casos mais graves (ou não), que os familiares conseguem perceber que não estão sozinhos e, consequentemente, podem aprender novas formas de adaptação.

O grupo funciona na sala de reunião da UTI, sob coordenação das psicólogas, com a presença do médico e, por vezes, de outro membro da equipe multidisciplinar (enfermeira, fisioterapeuta, nutricionista). O médico, após informar aos familiares o quadro clínico dos pacientes, em função de suas atividades, poderá ou não permanecer no grupo até o final do encontro.

É importante salientar que o grupo de suporte à família é aberto, pois a cada grupo novas famílias podem participar. Nos encontros, as dúvidas mais frequentes (relacionadas a procedimentos comumente efetuados) costumam ser sobre a traqueostomia e a gastrostomia e também sobre o "inchaço" apresentado por muitos pacientes (o que altera sua aparência, causando grande impacto nos familiares), além de questões ligadas à fisioterapia.

Com algumas interrupções, este grupo vem sendo realizado no CTI geral do HUPE aproximadamente há 12 anos. No momento não está ocorrendo, pois o mesmo encontra-se em reforma para ampliação de suas instalações; por este motivo a unidade está funcionando em local adaptado, no qual não há espaço físico apropriado que garanta a privacidade necessária para que os familiares sintam-se à vontade para trazer suas questões e manifestar suas emoções.

Consideramos de suma importância o trabalho de suporte psicológico aos familiares, pois estas pessoas, além de extremamente sofridas com a situação, têm muito a contribuir para a adaptação do paciente ao tratamento, funcionando como o elo entre o mundo externo do doente e o hospital e protegendo sua identidade fragmentada pela doença pela e internação. É incontestável que o familiar só poderá fornecer suporte adequado se tiver o apoio e o acolhimento necessários.

\section{O trabalho com a equipe da UTI}

A equipe de saúde também vivencia no seu cotidiano esse significado de viver e morrer, experimentando sentimentos ambivalentes de onipotência e impotência, sendo alvo de muitas cobranças por parte de todos os envolvidos e tendo que lidar com a percepção da própria finitude. O psicólogo deve atuar como facilitador do fluxo dessas emoções e reflexões, detectando os focos de estresse e sinalizando as defesas exacerbadas. ${ }^{18}$

A equipe deve estar preparada para lidar com sentimentos, emoções, valores morais e éticos, crença religiosa dos pacientes e familiares, de modo a respeitar e considerar as possíveis questões que emergirem. Este contato direto coloca os profissionais muitas vezes em situações que exigem um processo reflexivo sobre seus próprios valores, sua própria finitude e crença religiosa, bem como a angústia mediante a tomada de decisões.

Na UTI, um dos fatores adversos é a falta de tempo, o que dificulta o relacionamento interpessoal. Além disso, não é fácil para a equipe lidar com o sofrimento diário dos pacientes, familiares e com o seu próprio sofrimento. ${ }^{19}$

A comunicação entre o profissional e o 
doente, na maioria das vezes, não é clara. A dificuldade de falar por causa da cânula orotraqueal ou da traqueostomia torna-se mais um agravante, sendo necessária uma intervenção para auxiliar na comunicação. Uma boa estratégia é o atendimento conjunto com outros profissionais, promovendo um maior entendimento e melhor relacionamento, reduzindo desconfortos tanto da equipe como do paciente.

$\mathrm{Na}$ literatura atual, muitos estudos mencionam problemas frequentes relacionados à comunicação deficitária entre pacientes e a equipe de saúde. ${ }^{20}$ Quando há uma comunicação de qualidade entre profissional de saúde e paciente, este se sente mais motivado e encorajado a fazer perguntas, reduzindo seu sofrimento e ansiedade gerados pelo tratamento, além de se sentir mais satisfeito. ${ }^{21}$

É na prática do dia a dia que temos a oportunidade de aprender uns com os outros. Quando ocorre a mobilização da equipe em função de uma situação, seja com o paciente, seja com um familiar ou até mesmo com um membro da própria equipe, surge a oportunidade de trabalhar as questões emergentes. Por isso, é importante que o profissional de psicologia esteja atento e presente na UTI não só durante o período da visita, mas que ele participe das reuniões de equipe, permaneça na unidade após a saída dos familiares e participe do funcionamento de uma forma integral.

O trabalho do psicólogo é incessante e constitui um desafio, pois irá depender da demanda de cada profissional, que será diferente de acordo com o momento que estiver vivenciando; portanto, não existem regras rígidas. A forma de intervenção deverá ser criada, recriada e adaptada à medida que surgirem as necessidades de atuação da psicologia.

Quando o foco é a equipe, é importante não perder de vista o verdadeiro objetivo, que é a assistência. Um ambiente de trabalho com relações interpessoais adequadas torna-se mais agradável e produtivo, consequentemente gerando maior qualidade no atendimento aos pacientes e familiares.
Dentro de um trabalho grupal, o psicólogo precisa atuar como um agente facilitador do processo interativo, visando uma boa comunicação interpessoal.

Na tentativa de melhorar a comunicação e a relação entre todos os sujeitos envolvidos no CTI geral do HUPE, bem como discutir questões que permeiam as situações existentes em uma UTI, como a terminalidade, tivemos o ensejo de realizar palestras para a equipe multiprofissional, incluindo os residentes.

A oportunidade de a psicologia discutir com os profissionais através destes eventos permite uma melhor interação e troca de experiências entre os profissionais, ampliando ideias e promovendo reflexões importantes, favorecendo a resolução de problemas e ajudando na eficácia da comunicação.

O trabalho em equipe, principalmente dentro de uma instituição universitária, onde há um constante rodízio de estudantes, está sempre passando por modificações, o grupo está permanentemente em movimento, alterando sua dinâmica. Desta forma, o profissional de psicologia deve estar apto a lidar com as resistências e frustrações, sempre disposto a recomeçar.

A UTI, como ambiente de aprendizagem e atuação profissional, contribui, sobretudo, para o amadurecimento profissional e pessoal, uma vez que estimula o raciocínio clínico, conduz a uma reflexão crítica sobre conceitos e preconceitos relacionados com o viver e o morrer, a valores, à ética instrumental e dialógica, enfim às questões teórico-práticas da saúde e bioética no que se tem de mais diferencial e específico. ${ }^{22}$

\section{Conclusões}

A psicologia cumpre um relevante papel na UTI, visando a um atendimento que reconhece o paciente como um sujeito que possui desejos, medos e anseios, inserido em um contexto social, cultural e familiar com sua diversidade de aspectos psicológicos.

O trabalho com a família do paciente internado na UTI possibilita um lugar de escuta e intervenção em questões e mecanismos psi- 
cológicos que, se não trabalhados, poderão influenciar negativamente o paciente e sua doença.

No trabalho com a equipe, a psicologia fornece espaço para reflexão, suporte e apoio, favorecendo a percepção de questões que necessitam de elaboração e facilitando o fortalecimento dos vínculos entre o grupo de profissionais.

Enfim, o psicólogo funciona como facilitador das relações interpessoais dos sujeitos envolvidos, favorecendo uma comunicação mais eficaz, o que pode resultar em ambiente profissional mais agradável e melhor qualidade no atendimento aos pacientes e familiares.

\section{Referências}

1. Angerami-Camon VA. Tendências em psicologia hospitalar. 2. ed. São Paulo: Pioneira-Thomson Learning; 2004. p.1-29.

2. Pregnolatto AP, Agostinho VB. O psicólogo na unidade de terapia intensiva - Adulto. In: Batista MN , Dias RR, editores. Psicologia hospitalar: teoria, aplicação e casos clínicos. Rio de Janeiro: Guanabara Koogan;2003. p.93107.

3. Almeida EC. Psicólogo no hospital geral. Psicologia, Ciência e Profissão 2000;20(3):24-7.

4. Simão AT. Terapia Intensiva. Rio de Janeiro: Atheneu;1976. p.3-25.

5. Civetta J, Taylor R, Kirby R. Critical Care. Philadelphia: J.B. Lippincott Company;1988. p.247-54.

6. Mello FJ. Psicossomática hoje. Porto Alegre: Artes Médicas Sul:1992. p.353-83.

7. Ayres JRCM. Cuidado e reconstrução das práticas de saúde. Interface Comunicação Saúde e Educação 2004;8(14):73-92.

8. Campos TCP. Psicologia Hospitalar: a atuação do psicólogo em hospitais. São Paulo: EPU;1995. 112p.

9. Chiattone HBC, Sebastiani RW. A Ética em Psicologia Hospitalar. In: Angerami-Camon VA, organizador. A Ética na Saúde. São Paulo: Pioneira;1997.182p.

10. Ismael SMC. A inserção do psicólogo no contexto hospitalar. In: __. A prática psicológica e sua interface com as doenças. São Paulo: Casa do Psicólogo; 2005.p.17-36.

11. Simonetti A. Manual de Psicologia
Hospitalar: o mapa da doença. São Paulo: Casa do Psicólogo;2004. 201p.

12. Angerami-Camon VA, Trucharte FAR, Knijnik RB, Sebastiani RW, organizadores. Psicologia hospitalar: Teoria e prática. São Paulo: Pioneira;2006. p.15-28.

13. Rincon GH, Granados M, Unutzer J, Gomez M, Duran R, Badiel M, et al. Prevalence, detection na treatment of anxiety, depression and delirium in the adult critical care unit. Psychosomatics 2001;42(5):391-6.

14. Romano BW. Princípios para a prática da psicologia clínica em hospitais. São Paulo: Casa do Psicólogo;1999. p.115-67.

15. Oliveira EB, Sommerman RD. A família hospitalizada. In: Romano BW. Manual de Psicologia Clínica para Hospitais. São Paulo: Casa do Psicólogo;2008. p.217-23.

16. Botega NJ, Jacintho ACA, Damasceno BP, organizadores. Prática psiquiátrica no hospital geral: interconsulta e emergência. Porto Alegre: Artmed; 2006. p.395-404.

17. Santos FS. Cuidados Paliativos: discutindo a Vida, a Morte e o Morrer. São Paulo: Atheneu; 2009. p.223-44.

18. Angerami-Camon VA, organizador. Psicologia da Saúde: um novo significado para a prática clínica. São Paulo: Pioneira; 2002.p.215-25.

19. Leite DPM. Grupos em uma Unidade de Terapia Intensiva. In: Mello Filho J. Grupo e corpo. Porto Alegre: Artes Médicas; 2000. p.117-30.

20. Coyle N, Sculco L. Communication and the patient/physician relationship: a phenomenological inquiry. The Journal Of Supportive Oncology 2003 Set/Out [acesso 05 out 2012];1(3):206-15. Disponível em: http:// www.supportiveoncology.net/jso/journal/ articles/0103206.pdf

21. Savard M. Bridging the communication gap between physicians and their patients with physical symptoms of depression. Prim Care Companion J Clin Psychiatry 2004;6 (Suppl 1):17-24.

22. Pessini L, Barchifontaine CP. Dignidade e solidariedade no adeus à vida. In: Problemas atuais de bioética. 8. ed. São Paulo: Edições Loyola; 2008. p.198-201. 